\title{
The Immediate Effect of Interferential Current Therapy on Muscle Tone and Stiffness in Chronic Stroke Patients
}

\author{
Shin-Jun Park, PT, PhD • Kyun-Hee Cho, PT, MS ${ }^{1} \cdot$ Soon-Hee Kim, PT, $\mathrm{PhD}^{2 \dagger}$ \\ Department of Physical Therapy, GangDong University \\ ${ }^{1}$ Department of Physical Therapy, Graduate School, Yongin University \\ ${ }^{2}$ Department of Physical Therapy, Yongin University
}

Received: August 24, 2018 / Revised: August 27, 2018 / Accepted: September 21, 2018

(c) 2019 J Korean Soc Phys Med

\begin{abstract}
| Abstract |
Purpose: In chronic stroke patients, muscle tone and stiffness increase due to ankle spasticity. Electrotherapy may control the spasticity of patients with central nerve system damage via neurophysiological mechanisms. Therefore, this study was conducted to determine the immediate effects of interferential current therapy on gastrocnemius (GCM) muscle.
\end{abstract}

Methods: This study was a one-group pretest-posttest design and 20 stroke patients participated. The experimental group underwent interferential current therapy for GCM for 30 minutes. Muscle tone (MT) and stiffness were assessed using MYOTONER PRO. After 30 minutes of interferential current therapy, MT and stiffness of the affected side and unaffected side by GCM were measured.

Results: After interferential current therapy, the medial

†Corresponding Author : Soon-Hee Kim shkim2776@nate.com, https://orcid.org/0000-0001-5200-0051 This is an Open Access article distributed under the terms of the Creative Commons Attribution Non-Commercial License (http://creativecommons.org/licenses/by-nc/3.0) which permits unrestricted non-commercial use, distribution, and reproduction in any medium, provided the original work is properly cited.
GCM MT (Hz) was significantly reduced in stroke patients.

There was a significant difference in MT between affected GCM muscles and unaffected side medial GCM muscles before intervention, but there was no significant difference after interferential current therapy.

Conclusion: This study demonstrated that interferential current therapy had a positive effect, producing an immediate decrease in the medial GCM muscles tone of stroke patients. However, this study employed a one-group pretest-posttest design. Future studies will show differences in muscle tone compared to a control group or other electrical stimulation treatments.

Key Words: Asymmetrical muscle tone, Gastrocnemius muscle tone, Interferential current therapy, Stroke

\section{Introduction}

Following stroke, patients often experience asymmetrical muscle tone and stiffness in their hands[1] and legs[2] because of neurological impairment. An increase in gastrocnemius muscle (GCM) tone increases spasticity[3], whereas a decrease in GCM tone and stiffness may improve balance[4]. Therefore, many studies have investigated the 
reduction of GCM tone[2,4,5].

Several techniques that reduce muscle tone, such as robot-assisted training, mirror therapy[1], therapeutic exercise[2], and electrotherapy[4,5] have been investigated. Transcutaneous electrical nerve stimulation (TENS) and functional electrical stimulation (FES) reduce GCM tone and stiffness by stimulating the motor and sensory nerves using a low-frequency electrotherapy device[4]. While no studies have confirmed the immediate changes in muscle tone following electrotherapy, some have reported reductions in muscle tone at 4 weeks[4] and 6 weeks[6] after electrotherapy.

Interferential current therapy (ICT) is a pain-free electrotherapy that induces interference between currents of $4,000 \mathrm{~Hz}$ and $4,100 \mathrm{~Hz}$, thereby generating a beat frequency of $100 \mathrm{~Hz}$. A previous study found that the MAS score in stroke patients immediately decreased following ICT when compared to that of a control group[6]. However, in that study, electrotherapy was provided after therapeutic exercise. Furthermore, because the MAS score was used in the evaluation of the muscle, the functional state of the muscle could not be determined.

Therefore, this study was conducted to investigate immediate changes in GCM tone and stiffness in stroke patients following ICT in both affected and unaffected legs and to propose a new electrotherapy treatment.

\section{Methods}

\section{Participants}

Twenty patients with stroke from Y Rehabilitation Hospital, Gyeonggi-do, South Korea, were included in this study. The selection criteria were as follows: chronic stroke patients between 1 year or more and less than 3 years after onset, a Korean mini-mental state examination (K-MMSE) score of 24 or higher, a MAS score of 2 or higher of the affected GCM, and Brunnstrom stage 3 or higher[2]. All subjects participated in a discussion regarding
Table 1. Subject Characteristics

\begin{tabular}{lc}
\hline \multicolumn{1}{c}{ Classification } & Experimental group $(\mathrm{n}=20)$ \\
\hline Gender (male/female) & $17 / 3$ \\
Affected side (left/right) & $14 / 6$ \\
Disease duration (month) & $12.80 \pm 2.96$ \\
Age (years) & $64.00 \pm 7.81$ \\
Weight (kg) & $70.20 \pm 6.19$ \\
K-MMSE (point) & $26.30 \pm 1.41$ \\
\hline
\end{tabular}

Value are the means $\pm \mathrm{SD},{ }^{*} \mathrm{p}<.05, \mathrm{~K}$-MMSE:Korean version of the mini-mental state examination

the goals of this study and the precautions to be considered when using the electric equipment during treatment and provided written informed consent. This study was conducted in accordance with the Declaration of Helsinki.

\section{Measurement methods and device}

\section{1) Muscle tone (MT) and stiffness}

A MyotonPRO (Myoton AS, Estonia) system was used to evaluate MT and stiffness[2,4]. The subjects lay prone and were marked on the muscle belly. The idle vibration number of the equipment was set at 5 , and the equipment was positioned at a right angle to the marked muscle. After three repeated measurements, the average value was used as the result. The measured place was quiet and isolated space. The temperature of this space is $24^{\circ} \mathrm{C}$.

\section{Experimental design}

\section{1) Interferential current therapy}

The patients were subjected to electrotherapy intervention after they had undergone all treatment schedules in the physiotherapy room with no treatment schedules remaining. To eliminate the influence of the treatment on the intervention, the subjects underwent intervention after a break of 30 minutes. Four suction electrodes were attached to the muscle bellies of the lateral and medial GCM on 
Table 2. Changes in Gastrocnemius Muscle Tone

\begin{tabular}{cccc}
\hline & & Affected Side & Unaffected Side \\
\hline Medial & Before & $19.180 \pm .940$ & $18.140 \pm .460^{*}$ \\
Gastrocnemius $(\mathrm{Hz})$ & After & $18.700 \pm .840^{\ddagger}$ & $17.960 \pm .560$ \\
\hline Lateral & Before & $19.930 \pm .780$ & $18.330 \pm .550^{*}$ \\
Gastrocnemius $(\mathrm{Hz})$ & After & $20.090 \pm 1.160$ & $18.100 \pm .780^{*}$ \\
\hline
\end{tabular}

Values are the means \pm SD.

${ }^{*}$ Significant difference between affected side and unaffected side $(\mathrm{p}<.05)$.

${ }^{\ddagger}$ Significant difference before and after intervention $(p<.05)$.

the affected side. An interference aspiration low-frequency stimulator (ProMed III, STI-500, StraTek, Korea) was used for interferential current stimulation. In the subject group that underwent the stimulation, a quadrupole arrangement was used with four pads that were applied to the muscle belly surfaces of the ipsilateral medial and lateral GCM fibers. The amplitude-modulated beat frequency for the treatment site was set at $100 \mathrm{~Hz}$, the total treatment time was set at 30 minutes, the duty cycle was set at $50 \%$ and the actual stimulation was performed for 15 minutes[6].

\section{Statistical Analysis}

For all data analyses, SPSS 20.0 (Windows version) was used. General characteristics such as the average and standard deviation were evaluated using descriptive statistics. A paired-t test was used to compare the affected and unaffected sides before and after intervention. A $p<.05$ was taken to indicate statistical significance.

\section{Results}

\section{Comparison of MT before and after intervention}

There was no significant difference in the lateral GCM MT before and after ICT ( $p>05)$, but the medial GCM MT on the affected side decreased significantly after the intervention $(\mathrm{p}<.05)$. The MT of the medial and lateral GCM on the affected side before ICT were significantly different $(\mathrm{p}<.05)$ relative to the corresponding values on the unaffected side. However, there was no significant difference in the medial GCM MT between the affected and unaffected sides after ICT ( $p>.05)$. After the subjects were treated with ICT, no significant changes in the lateral GCM MT ( $>$.05) were noted; however, a significant decrease in the medial GCM MT on the affected side was observed after the intervention $(\mathrm{p}<.05)$. Before the intervention, there was a significant difference in MT of the medial and lateral GCM between the affected and unaffected sides $(p<.05)$. However, following the intervention, there was no significant difference in the medial GCM MT between the affected and unaffected sides $(\mathrm{p}>$.05).

\section{Comparison of muscle stiffness before and} after intervention

There was no significant difference in the medial and lateral GCM stiffness before and after ICT ( $p>05$ ). The stiffness values for the medial and lateral GCM on the affected side before ICT differed significantly $(p<.05)$ from those of the unaffected side. After the subjects were treated with ICT, significant changes in the medial and lateral GCM stiffness $(p<.05)$ were noted. Stiffness was unchanged in medial and lateral GCM. $(\mathrm{p}<.05)$.

\section{Discussion}

ICT is effective at decreasing the MAS score, which 
Table 3. Changes in Gastrocnemius Muscles Stiffness

\begin{tabular}{cccc}
\hline & & Affected side & Unaffected side \\
\hline Medial & Before & $359.220 \pm 28.620$ & $326.330 \pm 14.180^{*}$ \\
Gastrocnemius $(\mathrm{N} / \mathrm{m})$ & After & $349.000 \pm 23.920$ & $323.890 \pm 17.750^{*}$ \\
\hline Lateral & Before & $397.440 \pm 16.810$ & $353.220 \pm 19.630^{*}$ \\
Gastrocnemius $(\mathrm{N} / \mathrm{m})$ & After & $403.000 \pm 37.630$ & $348.670 \pm 31.650^{*}$ \\
\hline
\end{tabular}

Values are the means \pm SD.

*Significant difference between affected and unaffected side $(\mathrm{p}<.05)$.

‡Significant difference between before and after intervention $(\mathrm{p}<.05)$.

is an indicator of spasticity[6]. However, the present study is the first to use a MT measurement device to evaluate the tone and stiffness of the GCM following ICT. There was a significant decrease in the medial GCM tone after 30 minutes of ICT. Additionally, the medial GCM tone, which differentiated significantly compared to that on the unaffected side, showed no significant difference following ICT. Thus, symmetry in the medial GCM tone was confirmed.

The asymmetric flexor carpi radialis in stroke patients showed symmetry in MT and stiffness, and the stiffness of the flexor carpi ulnaris decreased significantly after mesh-glove electrical stimulation, robot therapy, therapeutic exercise, and mirror therapy[1]. The tone and stiffness of the medial GCM decreased after TENS and FES of the lower legs in stroke patients[4]. They noted that the absence of a significant difference following the intervention meant that normalization of muscle and decreased muscle tone were the results of functional state recovery[1,4].

Administration of ICT for 30 minutes after therapeutic exercise resulted in an immediate decrease in the MAS score, which was correlated with a reduction in the medial GCM tone[6]. An electric stimulation of $100 \mathrm{~Hz}$ isolates gamma-aminobutyric acid from the spinal cord dorsal horn[7]. In the present study, a $100 \mathrm{~Hz}$ current was applied using interfering middle frequencies ranging from 4,000 $\mathrm{Hz}$ to $4,100 \mathrm{~Hz}$. The electrical stimulation appeared to decrease muscle tone by increasing presynaptic inhibition [4]. Because the MAS score decreased by $41 \%$ after the application of middle frequency current, it would be more effective than TENS at reducing stiffness (TENS showed a $9-30 \%$ decrease in an earlier study)[6]. They further reported that, because the middle frequency current is associated with less skin resistance, it could penetrate the deep tissues effectively, thereby further reducing spasticity. However, it is difficult to say that ICT alone reduced the spasticity because subjects underwent ICT immediately after the therapeutic exercise in previous studies. However, in the present study, subjects underwent ICT after a break following completion of the scheduled therapeutic exercises. Thus, it was confirmed that 30 minutes of ICT decreased muscle tone, indicating symmetry in MT between the affected and unaffected sides.

It should be noted that this study could not determine the effectiveness of ICT because the number of subjects included was small, only those with high MAS scores were selected, and there was no control group. However, our finding that ICT alone, without therapeutic exercise, decreased the medial GCM tone is clinically significant. Further studies including more subjects and a control group should be conducted to clearly demonstrate the effects of ICT.

\section{Conclusion}

This study was conducted to investigate the immediate effects of interferential current therapy on the GCM muscle tone and stiffness of stroke patients. A total of 20 stroke 
patients were recruited with a one-group pretest-posttest design. Immediate reduction of the medial GCM tone in stroke patients after interferential current therapy application for 30 minutes was observed. In addition, the asymmetric medial GCM tone of the affected and the unaffected side became symmetric. Interferential current therapy reduced the medial GCM tone in stroke patients with spasticity in the lower leg. Therefore, interferential current therapy application to stroke patients with spasticity may help patients move their ankle.

\section{References}

[1] Bae SH, Lee JI, Kim KY, et al. Usefulness of Myotonometer for Measurement of Tissue Compliance on Medialis Gastrocnemius in Patients with Stroke. Journal of the Korea Academia-Industrial Cooperation Society. 2012;13(3):1129-37.

[2] Chuang LL, Wu CY, Lin KC, et al. Reliability, validity, and responsiveness of myotonometric measurement of muscle tone, elasticity, and stiffness in patients with stroke. Arch Phys Med Rehabil. 2012;93(3):532-40.

[3] Maeda Y, Lisi TL, Vance CGT, et al. Release of GABA and activation of GABA $A$ in the spinal cord mediates the effects of TENS in rats. Brain Res. 2007; 1136:43-50.

[4] Moon SH, Choi JH, Park SE. The effects of functional electrical stimulation on muscle tone and stiffness of stroke patients. J Phys Ther Sci. 2017;29(2):238-41.

[5] Park SJ, Cho KH, Cho YH. Effect of Exercise with Functional Electrical Stimulation and Transcutaneous Electrical Nerve Stimulation on Muscle Tone, Stiffness of Calf Muscle, and Balance Ability in Patients with Stroke. J Korean Soc Phys Med. 2017;12(2):43-52.

[6] Suh HR, Han HC, Cho HY. Immediate therapeutic effect of interferential current therapy on spasticity, balance, and gait function in chronic stroke patients: a randomized control trial. Clin Rehabil. 2014;28(9): 885-91.

[7] Wang JS, Lee SB, Moon SH. The immediate effect of PNF pattern on muscle tone and muscle stiffness in chronic stroke patient. J Phys Ther Sci. 2016;28(3):967-70. 
